J. Appl. Cryst. (1974). 7, 230

\title{
A Cold-Neutron Small-Angle Scattering (SAS) Device for Technological Applications
}

\author{
By P.Pizzi AND H. Walther \\ Fiat - Central Laboratories, Turin, Italy
}

\begin{abstract}
A cold-neutron SAS device to be operated in connexion with the $5 \mathrm{MW}$ Galileo Reactor in Pisa is briefly described. It consists of a liquid-propane cold-neutron source $\left(100^{\circ} \mathrm{K}\right)$; a neutron guide tube (mirror glass); a multidisc wavelength selector $(5 \leq \lambda \leq 20 \AA)$ whose resolution $(4 \leq \Delta \lambda / \lambda \leq 12 \%)$ can be varied according to the number of discs, and transmission increased by means of suitable guide tubes between the discs; a multicellular $\mathrm{BF}_{3}$ proportional counter connected with a minicomputer. Some preliminary results have been obtained in spite of provisional wavelength-selecting and counting systems; they refer to some technological problems: scattering variations in $\mathrm{Cu}, \mathrm{Ni}, \mathrm{Fe}$ have been measured as produced by recrystallization and cold working. An increase of scattering from Inconel X-750 turbine blades has been observed as a function of operation time. Furthermore, scattering variations as a consequence of age-hardening treatments have been measured in the same alloy. Finally a scattering effect has been observed in various metals after exposure to atomic hydrogen, which might be important for investigating the problem of hydrogen embrittlement. According to those results (although only partially interpreted), a number of interesting possibilities are indicated, where neutron smallangle scattering may be applied in technology for non-destructive testing.
\end{abstract}

J. Appl. Cryst. (1974). 7, 230

\section{Superconductivity and Correlation of Magnetic Moments in $\mathbf{C e}_{1-x} \mathbf{T b}_{x} \mathbf{R u _ { 2 }}$}

\author{
By S. Roth, K. Ibel and W. Just \\ Institut Laue-Langevin, B.P. 156, 38042 Grenoble Cedex, France
}

(Received 14 September 1973; accepted 4 October 1973)

\begin{abstract}
The correlation of magnetic moments has been investigated by neutron small-angle scattering in superconducting and normal $\mathrm{Ce}_{1-x} \mathrm{~Tb}_{x} \mathrm{Ru}_{2}$. Samples with $20 \% \mathrm{~Tb}$ are superconducting below $2 \cdot 6^{\circ} \mathrm{K}$. In these samples ferromagnetic short-range order is found below $10^{\circ} \mathrm{K}$. In spite of a maximum in the static susceptibility at $5^{\circ} \mathrm{K}$ no transition to long-range order is observed. In the whole temperature range between 1.5 and $10^{\circ} \mathrm{K}$ the experimental data can be fitted by an Ornstein-Zernike correlation function. At $1.5{ }^{\circ} \mathrm{K}$ the correlation length is about $15 \AA$. No significant change is observed at the superconductive transition temperature. Samples with $40 \% \mathrm{~Tb}$ are not superconducting. The static susceptibility shows a maximum at $13^{\circ} \mathrm{K}$. Above this temperature again ferromagnetic short-range order of the Ornstein-Zernike type is found. Below $13^{\circ} \mathrm{K}$ the intensity of scattered neutrons drops faster with scattering angle indicating long-range order.
\end{abstract}

\section{Introduction}

Superconductors containing magnetic impurities have been the object of many investigations in the last decade. Special attention has been paid to those systems where even a fairly high impurity concentration leads to only a slight decrease in the superconducting transition temperature. Here ordering effects of the impurity spins become important and the coexistence of ferromagnetism and superconductivity has been predicted (Matthias, Suhl \& Corenzwit, 1958). Typical systems of this kind are mixed crystals of a superconducting and a ferromagnetic component, such as the rare-earth Laves phases.
Usually the magnetic properties are studied by the investigation of the macroscopic susceptibility (Hillenbrand \& Wilhelm, 1970). Below the superconducting transition temperature this method is no longer appropriate since the sample becomes diamagnetic because of the shielding currents. The measurements of specific heat (Rupp, 1973) and of Mössbauer line broadening (Taylor et al., 1972) (Steiner, Gumprecht \& Hüfner, 1973) yield further information, but the most direct way of studying magnetic ordering is neutron scattering.

For the present investigation the system $\mathrm{Ce}_{1-x} \mathrm{~Tb}_{x} \mathrm{Ru}_{2}$ has been chosen, because this system has already been studied by other methods (Hillenbrand \& Wilhelm 